\title{
Forecasting INR Exchange Rate Against USD, GBP, JPY, SGD, EUR, AED Using Machine Learning
}

\author{
${ }^{1}$ Sumith Pevekar \\ ${ }^{1}$ Department Of Computer Engineering, Vidyalankar Institute Of Technology, Mumbai, Maharashtra, \\ India
}

Article DOI: https://doi.org/10.36713/epra7960

DOI No: 10.36713/epra7960

\begin{abstract}
The price of a native currency expressed in terms of another currency is known as a foreign exchange rate. In other terms, a foreign exchange rate compares the value of one currency to that of another. The value of standardized currencies varies with demand, supply, and consumer confidence around the world due to which their values fluctuate over time. To forecast the exchange rate of INR, I have developed a machine learning model. The model was trained to estimate six foreign currency exchange rates against the Indian Rupee using historical data. This model uses Random Forest algorithm to train and predict the values. The suggested system's predicting performance is assessed and contrasted using statistical metrics. According to the findings, the Random Forest algorithm-based model predicts well and achieves an accuracy of $93.61 \%$.
\end{abstract}

KEYWORDS: Regression, Random Forest, Exchange Rate, INR

\section{INTRODUCTION}

The value of one country's currency in relation to the currency of another country or economic zone is known as an exchange rate. There are various types of exchange rates out of which free floating exchange rate is the one whose exchange rate rises and falls due to changes in the foreign exchange market. Economists and investors routinely estimate future exchange rates to determine monetary worth based on their projections.

There is no single currency system that is appropriate for all countries or at all periods [1]. Meese and Rogoff's landmark work (1983) demonstrated that econometric specifications based on macroeconomic fundamentals cannot beat basic random walk forecasts over short time horizons [2]. Various models are used to forecast a currency's future exchange rate. The exchange rate also depends on sentiments. The exchange rates are intrinsically noisy, non-stationary, and deterministically chaotic and predicting them is one of the difficult tasks [3]. The country's currency depreciation or appreciation rate is influenced by

factors such as people's purchasing power, migration, commodity trades, and labor force, all of which have a significant impact on international exchanges [4]. Because of market strategy and investor interest in international enterprises, exchange rate forecasting is critical in today's world economy [5].

The main goal of this research is to present a Random Forest based model for predicting USD, JPY,SGD, EUR, GBP, AED exchange rates versus the Indian Rupee. In this work, I collected and used 875 historical exchange rates data from year 2016 to 2021 as inputs to create the prediction model, with an additional 431 exchange rates data used to test the model. Several studies have been conducted in many countries to forecast exchange rates however no 


\section{EPRA International Journal of Research and Development (IJRD) Volume: 6 | Issue: 7 | July 2021 \\ - Peer Reviewed Journal}

published research on Indian Rupee against vast range of exchange rates has been discovered.

\section{RELATED WORK}

While working on the project, I found a lot of applications that shared goal with this project.

The exchange rates depend upon various factors. But it is more dependent on the trades between the two countries. The terms of trade of a country have improved if the price of its exports grows faster than the price of its imports. The terms of trade affect the exchange rate considerably. Currency exchange rates are also affected by changes in market inflation. The value of a country's currency will appreciate if its inflation rate is lower than that of another. There are many factors which affect exchange rates but forecasting the exchange rate based on historical data is the only method to predict future rate price.

Venugopalsanke and Raghavender Mamillapally (2014) investigated the out-of-sample forecast performance of auto regressive integrated moving average (ARIMA) and Feed forward neural networks (FFNN) models for forecasting INR/USD exchange rate [6]. According to them, The ARIMA model predicts exchange rates in a linear fashion, but the FFNN model displays changing exchange rates based on the preceding observation's input. In comparison to the ARIMA model, these projections demonstrate that the FFNN model accurately predicts future exchange rates with the least amount of error.

Yusuf Perwej and Asif Perwej (2012) examined the effects of neural network parameters on neural network modelling and forecasting performance, both in-sample (training set) and outof-sample (test set) results [7]. They concluded that it is not reasonable to evaluate the Artificial Neural Network capabilities solely on the basis of the training sample for forecasting purposes. There are no widely acknowledged approaches for building the optimal prediction model with only in-sample training data. The best network architecture should be chosen based on test sample results.

\section{METHODOLOGY}

\subsection{Data Collection}

The dataset for this model is downloaded from yahoo finance which provides access to the historical values of USD/INR, JPY/INR, GBP/INR, SGD/INR, EUR/INR, AED/INR exchange rates. The dataset contains the price values of last five years.
The downloaded data gets stored in the form of csv file. The dataset is then read and converted in the form of Pandas data frame.

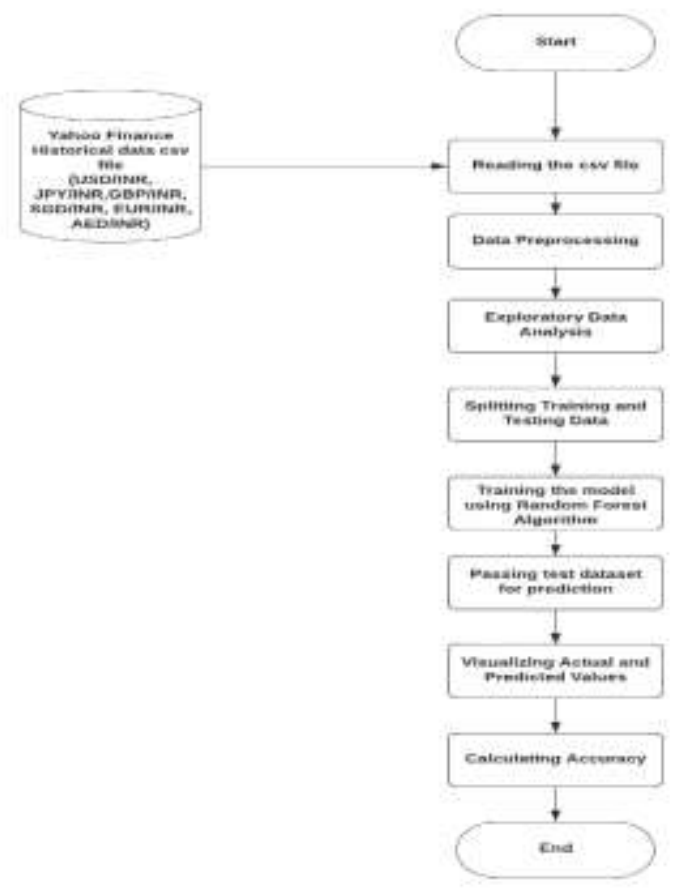

Figure 3.1 Proposed Workflow

\subsection{Exploratory Data Analysis}

The six datasets taken for the development of the model contain $\mathrm{NaN}$ and infinity values. The presence of these values may lower the accuracy of the model. In data preprocessing, the non-required values present in the dataset is removed. The resultant data is free from noise which helps in better training of the model. This helps in getting better predictions, thereby increasing the accuracy of the model. The total number of $\mathrm{NaN}$ and infinite values are shown in fig 3.2.

Total nunber of Nall and w values in INR/USD dataset: 138 Total nurber of Nall and o values in INR/JPY dataset: 138 Total nurber of Nall and w values in INR/SGD dataset: 138 Total nurber of Nall and $\infty$ values in INR/AED dataset: 120 Total nurber of Nall and wo values in INR/EUR dataset: 132 Total nurber of Nall and so values in IMR/GBP dataset: 132

Figure 3.2 Total number of NaN and Infinity values in dataset 


\section{EPRA International Journal of Research and Development (IJRD) Volume: 6 | Issue: 7 | July 2021 \\ - Peer Reviewed Journal}

\subsection{Data Processing and Cleaning}

The original dataset contained lot of null values as evident from fig 3.2. These values were removed from all the datasets. The USD/INR dataset aft

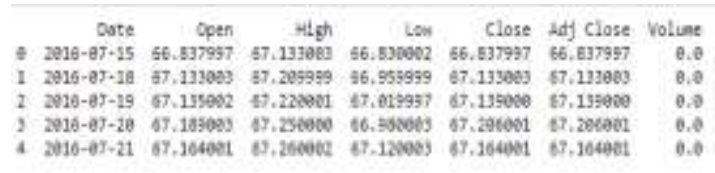

Figure 3.3 USD/INR dataset

\subsection{Data Visualization}

The closing price of each currency is plotted against the number of days using matplotlib.pyplot library. By looking at the plots, I discovered that the data values follow a trend. Exchange rates shows a predictable pattern of falling prices and rising prices, or vice versa.

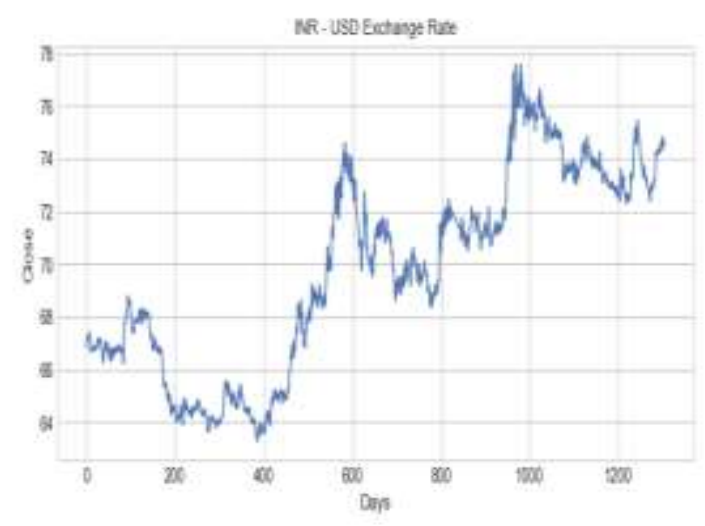

Figure 3.4 INR-USD (close vs no. of days)

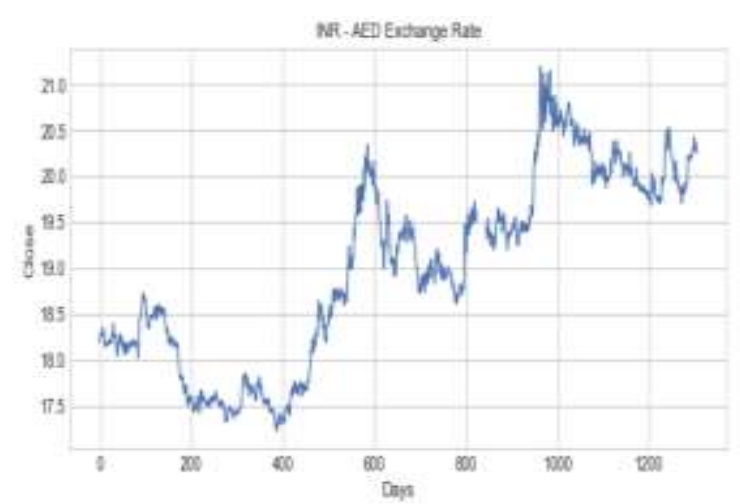

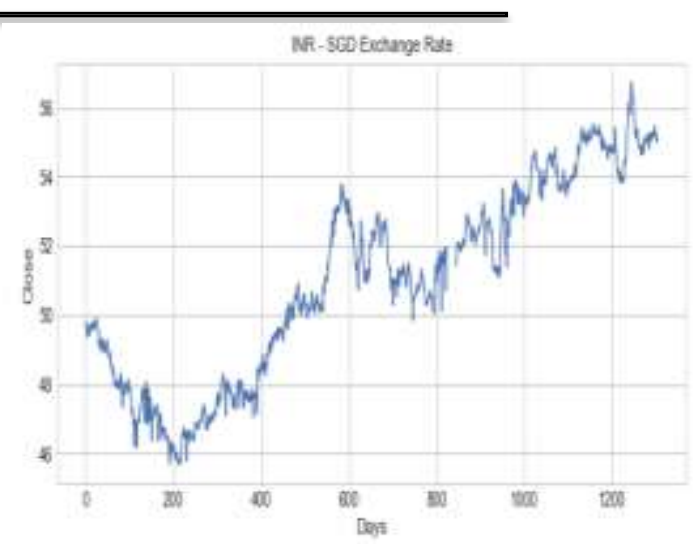

Figure 3.6 INR-SGD (close vs no. of days)

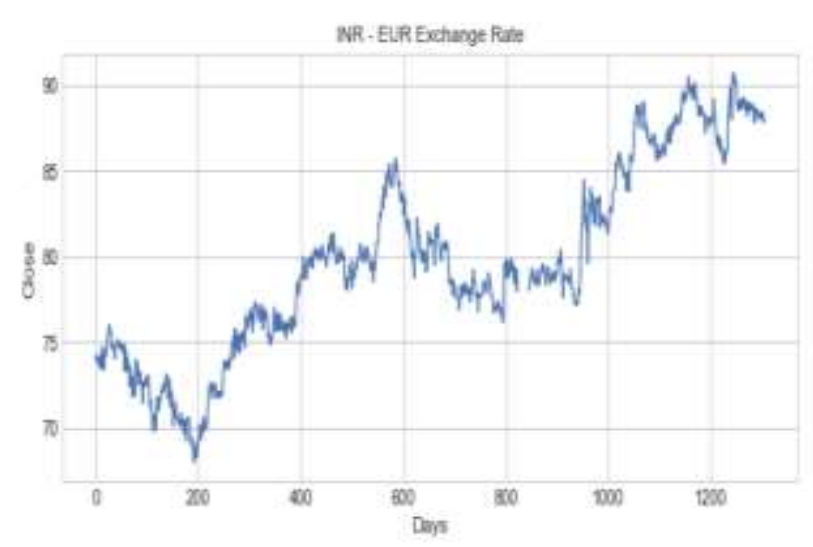

Figure 3.7 INR-EUR (close vs no. of days)

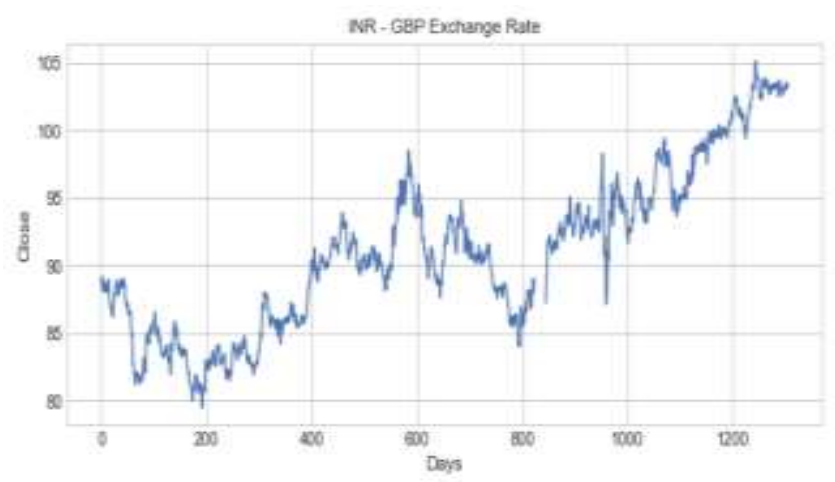

Figure 3.8 INR-GBP (close vs no. of days)

Figure 3.5 INR-AED (close vs no. of days) 


\section{EPRA International Journal of Research and Development (IJRD)}

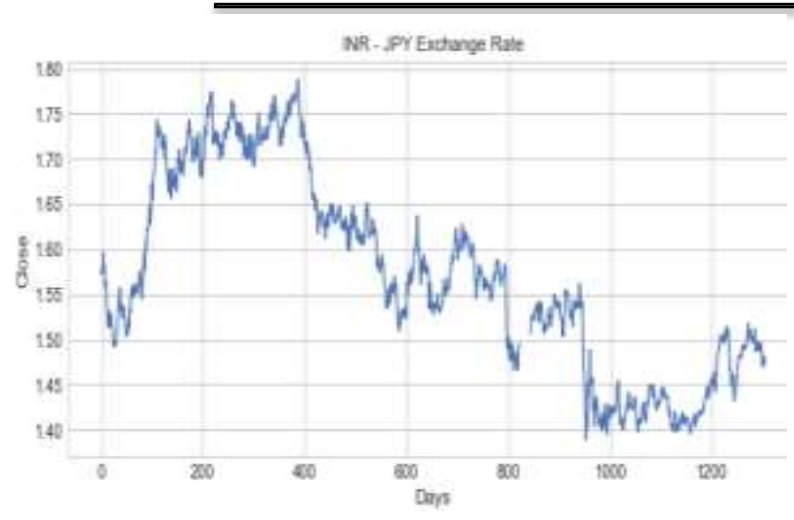

predicts the INR accurately against the exchange rates. The result of model is plotted in the form of actual and predicted values and is shown in figure $4.1,4.2,4.3,4.4,4.5,4.6$.

Figure 3.9 INR-JPY (close vs no. of days)

\subsection{Random Forest}

Random Forest is a tree-based system that makes decisions based on the characteristics aspects of numerous Decision Trees. In my model, I have used the random forest as the algorithm to predict the test values. Since Random Forest Regression is a supervised machine learning algorithm, it needs to be trained beforehand to make accurate predictions. I have trained the model using training data values and then passed the test data values to make the predictions. The working of Random Forest is shown in fig 4.0.
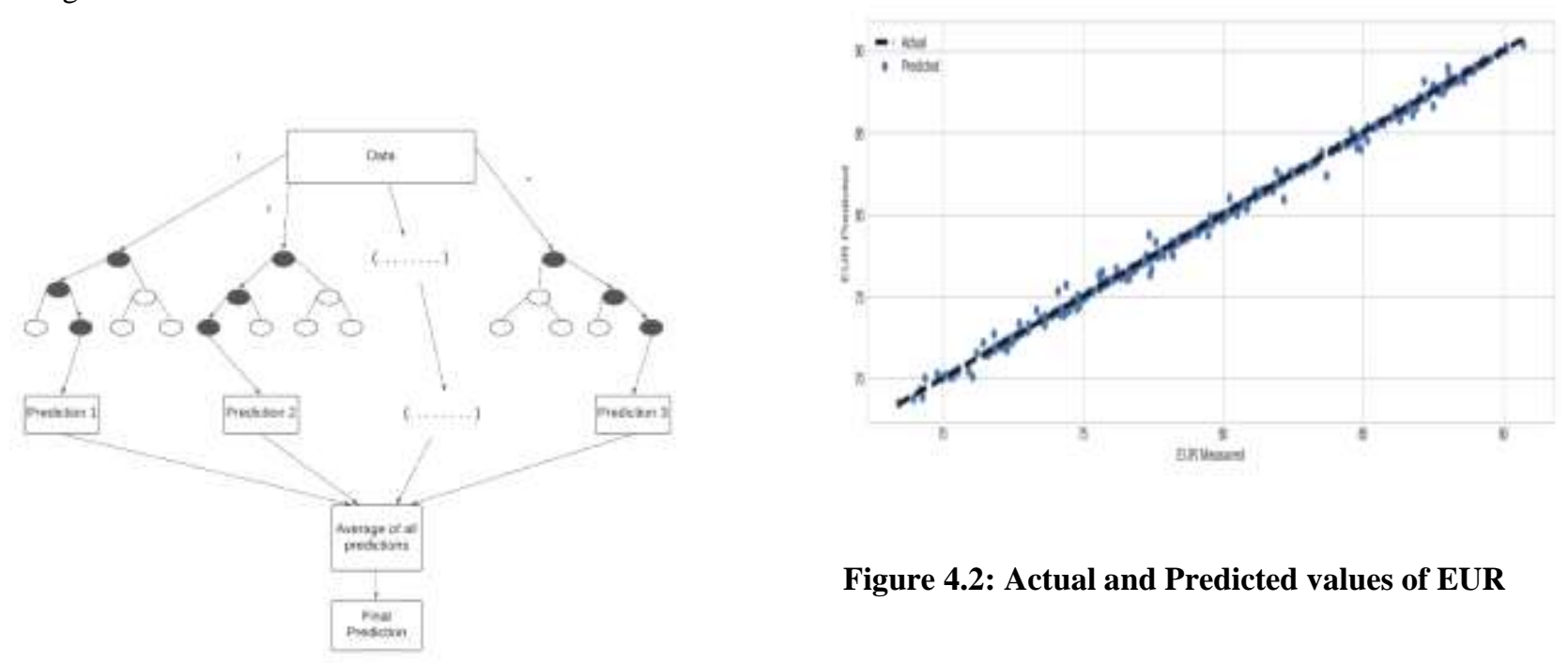

Figure 3.10: Random Forest

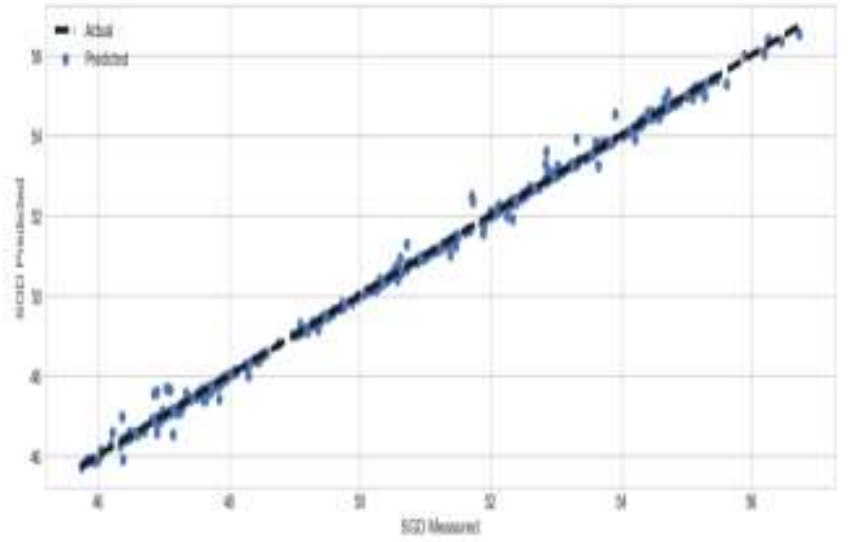

Figure 4.1: Actual and Predicted values of SGD

Figure 4.2: Actual and Predicted values of EUR

\section{RESULTS}

The model was trained and tested on datasets of various exchange rates. It was found that the model 


\section{EPRA International Journal of Research and Development (IJRD)

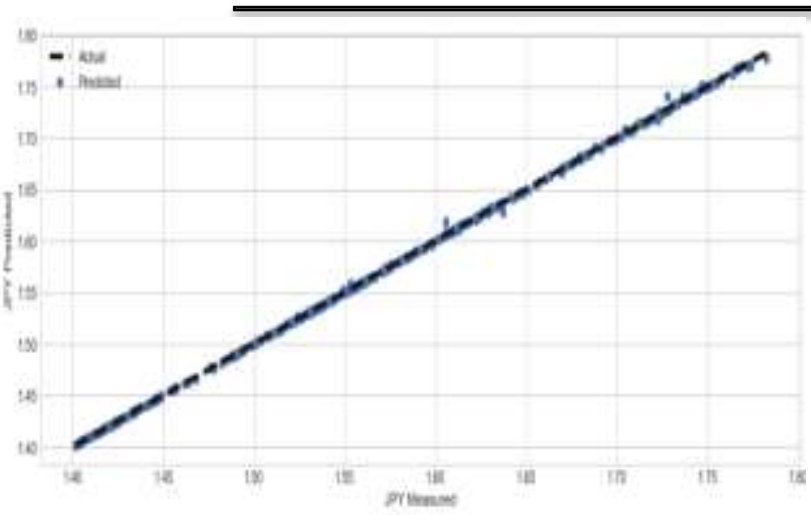

Figure 4.3: Actual and Predicted values of JPY

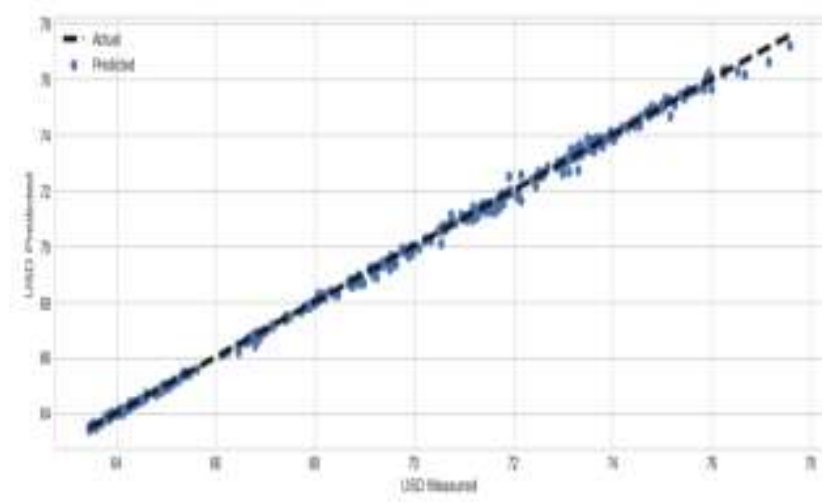

Figure 4.4: Actual and Predicted values of USD

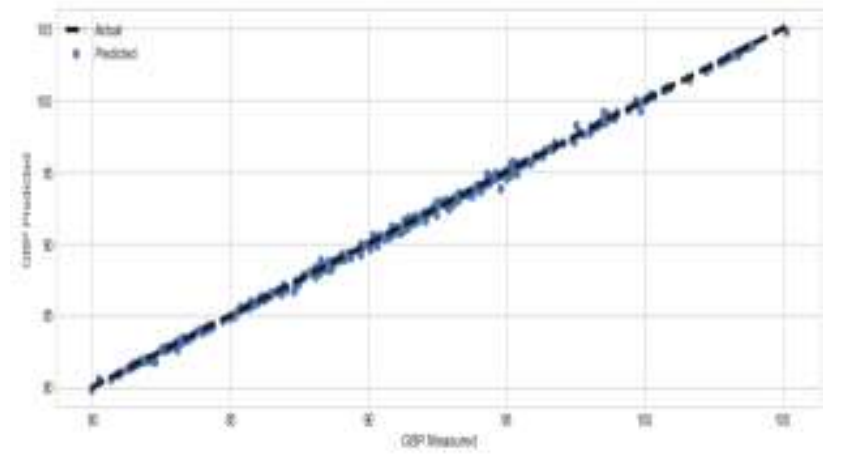

Figure 4.5: Actual and Predicted values of GBP

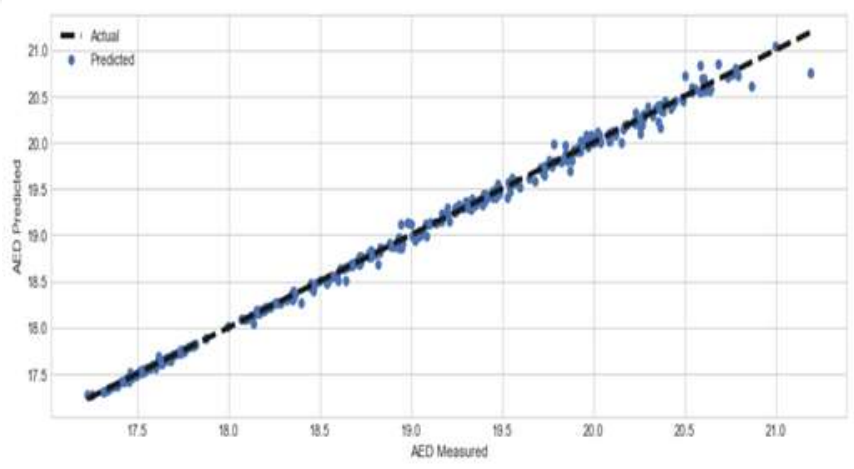

Figure 4.6: Actual and Predicted values of AED The model achieves an overall accuracy of $93.61 \%$. with a mean absolute error as 4.45 degrees.

Metrics for Random Forest Trained on Original Data Average absolute error: 4.45 degrees.

Accuracy: $93.61 \%$.

\section{CONCLUSION AND FUTURE SCOPE}

In this research, a machine learning model was implemented to forecast INR exchange rate against the USD, JPY, SGD, AED, GBP, EUR. The fundamental feature of the proposed work is the machine learning model when provided with the test values almost accurately predicts the rates as the actual values. The model used Random Forest Regression to carry out the desired predictions. It was found that the used algorithm was able to meet the requirements and can be very well used to do the predictions. The key shortcoming is that the model may be prone to the negative news affecting the exchange rates considerably.

In future, this model can be employed and used to take key decisions related to the trading of stocks as well as for the accumulation of long term stocks. The model can also be used to determine the relative level of economic health of a particular country.

\section{REFERENCES}

1. Meese, R. A., \& Rogoff, K. (1983). Journal of International Economics, 14, pp: 3-24.

2. Frankel, J. A. (1999). Cambridge, Massachusetts: National Bureau of Economic Research, NBER Working Paper: 7858

3. N.V. CHANDRASEKARA \& C.D. TILAKARATNE, Forecasting Exchange Rates using Artificial Neural Networks, ResearchGate, January 2009. 


\section{EPRA International Journal of Research and Development (IJRD)}

Volume: 6 | Issue: 7 | July 2021

- Peer Reviewed Journal

4. Taher Ali Kapadiya, OSR Journal of Business and Management (IOSR-JBM, Volume 20, Issue 9. Ver. VI (September. 2018), pp: 57-61

5. Subhasmit Mondal, IOSR Journal of Computer Engineering (IOSR-JCE), Volume 17, Issue 2, Ver. VI (Mar-Apr. 2015), pp: 01-10

6. VenugopalSanke, Raghavender Mamillapally, Forecasting of INR/USD Exchange Rates, International Journal of Mathematical Sciences, Technology and Humanities 114 (2014), pp: $1228-1235$

7. Yusuf Perwej, Asif Perwej, Forecasting of Indian Rupee (INR)/US Dollar (USD) Currency Exchange Rate Using Artificial Neural Network, (IJCSEA) Vol.2, April 2012, pp: 41 -52 